\title{
Short- and long-term outcomes as well as anal function of transanal natural orifice specimen extraction surgery versus conventional laparoscopic surgery for sigmoid colon or rectal cancer resection: a retrospective study with over 5-year follow-up
}

\author{
Jianlu Zhang ${ }^{1}$, Weiqing $\mathrm{Li}^{2}$, Ying $\mathrm{Li}^{1}$, Buhe Amin ${ }^{1}$, Nengwei Zhang ${ }^{1}$, Zhipeng Sun ${ }^{1}$, Bin Zhu ${ }^{1}$ \\ ${ }^{1}$ Department of General Surgery, Beijing Shijitan Hospital, Capital Medical University, Beijing, China \\ ${ }^{2}$ Department of Thoracic Surgery, Beijing Shijitan Hospital, Capital Medical University, Beijing, China
}

Videosurgery Miniinv 2022; 17 (2): 344-351

DOI: https://doi.org/10.5114/wiitm.2022.113567

\begin{abstract}
Introduction: To date, long-term safety including functional outcomes of transanal natural orifice specimen extraction surgery (NOSES) for colorectal cancer resection has not been confirmed.

Aim: To explore the short- and long-term outcomes as well as anal function of transanal NOSES versus conventional laparoscopic surgery for sigmoid colon or rectal cancer resection.

Material and methods: A retrospective review of data from a prospectively maintained database was performed to analyze the data of 69 patients who underwent transanal NOSES for sigmoid colon or rectal cancer resections and another 69 matched patients who underwent conventional laparoscopic (CL) surgery. Anal function of patients was evaluated using the Wexner fecal incontinence scale postoperatively.

Results: Transanal NOSES resulted in faster recovery of intestinal function, shorter postoperative length of stay, less incisional pain, fewer postoperative complications and shorter scars than CL surgery $(p<0.05)$. The two groups had similar overall survival $(p=0.863)$ and disease-free survival $(p=0.961)$. Wexner scores of the NOSES group at 1,3 and 6 months after surgery were higher than in the CL group $(p<0.05)$, and there was no difference between the two groups at 12, 18 and 24 months after surgery.

Conclusions: Transanal NOSES achieves similar survival outcomes to CL surgery. Transanal NOSES has the advantages of faster recovery, shorter postoperative hospital stay, less incisional pain, shorter scars, etc. However, transanal NOSES can indeed impair anal function, needing more attention.
\end{abstract}

Key words: colorectal cancer, laparoscopic surgery, long-term follow-up, natural orifice specimen extraction surgery, anal function.

\section{Introduction}

Natural orifice specimen extraction surgery (NOSES) in colorectal cancer has been explored in many clinical studies [1, 2]. Compared with conventional laparoscopic (CL) surgery, NOSES reduces assisted incision and related postoperative pain and incisional complications as well as obtaining faster recovery and better cosmetic effects $[3,4]$. However, few studies showing long-term results of NOSES for colorectal cancer resection have been reported, and the long-term safety of NOSES has not been fully proven [5-7]. 
Anal damage may occur during the removal and anastomosis of the intestinal tube through the anus $[3,8,9]$. In practice, many patients refused transanal NOSES out of the concern about the risk of fecal incontinence. Besides oncological outcomes, the safety of the new technique should include the functional safety. To date, no long-term dynamic assessment of anal function after NOSES colorectal resection has been reported.

\section{Aim}

Therefore, we completed a retrospective study with up to 112 months follow-up to explore the long-term outcomes of transanal NOSES and CL sigmoid colon and rectal cancer resection. Also, the anal function after surgery was evaluated using the Wexner fecal incontinence grading scale dynamically, which consists of five questions and the total score ranging from 0 (perfect continence) to 20 (total incontinence) $[10,11]$.

\section{Material and methods}

\section{Patients}

A retrospective review of data from a prospectively maintained database of patients underwent transanal NOSES for sigmoid colon or rectal cancer resections between January 2012 and May 2016 was performed. The exclusion criteria included: 1) the tumor was over $6 \mathrm{~cm}$ in diameter; 2) acute abdomen, including intestinal obstruction and perforation; 3) patients in American Joint Committee on Cancer (AJCC) pTNM Staging IV; 4) body mass index (BMI) $>35 \mathrm{~kg} / \mathrm{m}^{2}$; 5) lower margin of a tumor $<5 \mathrm{~cm}$ from the anal verge; 6) incomplete data. Based on the prospectively maintained database, 69 patients who underwent transanal NOSES sigmoid colon or rectal colon cancer resection were identified and allocated to the NOSES group. In order to reduce the selection bias, these 69 patients were matched individually by age, gender, BMI, neoadjuvant therapy, tumor location, tumor size, tumor differentiation, PTNM stage, and history of abdominal surgery in the same period with 69 patients who underwent CL sigmoid colon or rectal colon cancer resection, and these patients were allocated to the $\mathrm{CL}$ group.

In addition to the above matching information, data were collected for the two groups including operation related variables, postoperative variables and follow-up data. The pain scores of the two groups of patients measured using the numerical rating scale (NRS) from 0 to 10 on the first, third and seventh days after surgery were collected. The Wexner fecal incontinence grading scale [11] (Table I) was used to evaluate the anal function of patients dynamically at 1 month, 3 months, 6 months, 12 months, 18 months and 2 years after surgery through mail, email or social software. Perianal operation history and obstetric history, two factors that may affect anal function, were also determined [12]. This study has been approved by the Ethics Committee of Beijing Shijitan Hospital (2012000019), and written informed consent was obtained from all patients.

\section{Operation}

All procedures were performed by the surgeons of one team in our department who had extensive experience in laparoscopic colorectal surgery. All patients were administered polyethylene glycol electrolyte powder (Compound Macrogol 4000 Powder for Oral Solution) for intestinal preparation one day before the operation. Venous-inhalation combined general anesthesia was used in all the patients. In general, cephalosporins were used in every patient $30 \mathrm{~min}$ before operation, while aminoglycosides were used in those patients with cephalosporin allergy.

Table I. Wexner fecal incontinence scoring system [11]

\begin{tabular}{|lccccc|}
\hline Type of incontinence & \multicolumn{5}{c|}{ Frequency } \\
\cline { 2 - 6 } & Never & Rarely & Sometimes & Usually & Always \\
\hline Solid & 0 & 1 & 2 & 3 & 4 \\
\hline Liquid & 0 & 1 & 2 & 3 & 4 \\
\hline Gas & 0 & 1 & 2 & 3 & 4 \\
\hline Wears pads & 0 & 1 & 2 & 3 & 4 \\
\hline Lifestyle alteration & 0 & 1 & 2 & 3 & 4 \\
\hline
\end{tabular}

Never -0 , Rarely $\leq$ once/month, once/month $\leq$ Sometimes $\leq$ once/week, once/week $\leq$ Usually $\leq$ once/day, Always $\geq$ once/day. 
The patient was placed in the traditional lithotomy position with pneumoperitoneum pressure maintained at 12-14 mm Hg. The operation was performed according to different diseases and locations of tumors. General principles of total mesorectal excision (TME) or complete mesocolic excision (CME) and functional protection were followed, including the "no-touch isolation" technique, en bloc resection of the tumor combined with the surrounding involved tissues, sufficient length of negative margins, thorough lymph node dissection and careful abdominal lavage. In the NOSES group, the specimen was pulled out through the anus and in the CL group the specimen was removed through an assisted abdominal incision on the mid-line. Patient-controlled analgesia was used for all the patients after surgery. Intraoperative evaluation indices included operative time, blood loss, intraoperative complications, number of harvested lymph nodes, and the integrity of the specimen.

\section{Follow-up}

Every patient underwent long-term follow-up by reexamination and telephone interview. Follow-up was in accordance with the guidelines of the $\mathrm{Na}$ tional Comprehensive Cancer Network and adjusted according to patients' will, including: 1) physical examination, carcinoembryonic antigen (CEA), carbohydrate antigen 19-9 (CA-199) every 3-6 months for the first 3 years, then every 6 months from the third year; 2) chest, abdominal and pelvic computed tomography (CT) or magnetic resonance imaging (MRI) once a year in the first 5 years; 3 ) colonoscopy within 1 year, 3 years and five years after the operation. The long-term endpoints of this study were 5-year and 8-year overall survival (OS) and disease-free survival (DFS). According to the guidelines of the National Comprehensive Cancer Network, all patients with T3/T4 or positive lymph nodes underwent adjuvant chemotherapy and there was no bias in chemotherapy between the two groups.

\section{Ethics approval and consent to participate}

This study has been approved by the Ethics Committee of Beijing Shijitan Hospital (2012000019), and written informed consent was obtained from all patients. All procedures performed were in accordance with the ethical standards of the institution and with the 1964 Helsinki Declaration and its later amendments or comparable ethical standards.

\section{Statistical analysis}

Statistical analysis was performed using SPSS 22.0 statistical software (SPSS Inc., Chicago, Illinois, USA). Continuous variables were presented as mean \pm standard deviation (SD) and compared using Student's $t$-test or the Wilcoxon rank-sum test. Categorical variables were presented as numbers (percentages) and compared using the $\chi^{2}$ test or Fisher's exact test. Kaplan-Meier plots and log-rank tests were used for survival analyses. $P$-values were derived from either one-tailed or two-tailed tests, and $p<0.05$ was set as the threshold for statistical significance.

\section{Results}

One hundred and thirty-eight patients were included in this study. The clinical and pathological characteristics of the two groups were comparable $(p>0.05)$ (Table II). Operation related variables are presented in Table III. The operation time in the NOSES group was significantly longer than that in the $\mathrm{CL}$ group $(241.18 \pm 75.57$ vs. $202.51 \pm 71.24 \mathrm{~min}$, $p<0.001)$. The blood loss and number of harvested lymph nodes were similar between the two groups $(p=0.301$ and $p=0.721$, respectively). There was no positive margin or tumor rupture in the two groups. Table IV presents the postoperative evaluation. The recovery time of bowel function was $3.21 \pm 1.38$ days in the NOSES group, which was significantly shorter than $4.35 \pm 1.46$ days in the CL group. Postoperative length of stay in the NOSES group was also significantly shorter than that in the CL group (10.18 \pm 3.27 vs. $13.65 \pm 5.69$ days, $p<0.001$ ). The complication rate in the $\mathrm{CL}$ group was higher than that of the NOSES group ( $13.04 \%$ vs. $33.33 \%, p=0.047)$. In particular, none of the patients in the NOSES group had incisional infection, whereas 6 cases occurred in the $\mathrm{CL}$ group $(p=0.028)$. In the NOSES group, pain scores on 1 day and 3 days after surgery were 3.91 \pm 1.63 and $2.64 \pm 1.35$, which were significantly lower than $5.09 \pm 1.92$ and $3.71 \pm 2.62$ in the CL group ( $p<$ 0.001 ). Regarding the postoperative cosmetic result, the total length of scars in the NOSES group was significantly shorter than that in the $\mathrm{CL}$ group (4.17 \pm 1.25 vs. $9.05 \pm 3.24 \mathrm{~cm}, p<0.001$ ).

Table $V$ presents the anal function related data. The Wexner scores at 1 month, 3 months and 6 months after surgery were $2.57 \pm 3.05,1.72 \pm 1.92$ and $1.28 \pm 1.62$ in the NOSES group respectively, sig- 
Table II. Clinical and pathological characteristics

\begin{tabular}{|lccc|}
\hline Parameter & NOSES $(n=69)$ & $\mathrm{CL}(n=69)$ & $P$-value \\
\hline Age $[$ years] & $54.42 \pm 11.56$ & $55.28 \pm 11.49$ & 0.502 \\
\hline Gender (male/female) & $37 / 32$ & $37 / 32$ & 1.000 \\
\hline BMI $\left[\mathrm{kg} / \mathrm{m}^{2}\right]$ & $23.27 \pm 3.29$ & $23.18 \pm 3.31$ & 0.882 \\
\hline Neoadjuvant therapy $[n(\%)]$ & $5(7.25)$ & $5(7.25)$ & 1.000 \\
\hline Tumor location $[n(\%)]:$ & & 0.805 \\
\hline Sigmoid & $28(42.03)$ & $27(39.13)$ & 0.567 \\
\hline Rectum & $41(59.42)$ & $42(60.87)$ & 0.819 \\
\hline Tumor diameter $[\mathrm{cm}]$ & $3.14 \pm 1.48$ & $3.18 \pm 1.53$ & \\
\hline Differentiation $[n(\%)]:$ & & $19(27.54)$ & 0.735 \\
\hline Well & $21(30.43)$ & $44(63.77)$ & $6(8.70)$ \\
\hline Moderate & $43(62.32)$ & & \\
\hline Poor & $5(7.25)$ & $20(28.99)$ & 0.425 \\
\hline PTNM stage $[n(\%)]:$ & $18(26.09)$ & $24(36.23)$ & $7(10.14)$ \\
\hline I & $24(34.78)$ & $27(39.13)$ & \\
\hline II & $5(7.25)$ & & \\
\hline III & & & \\
\hline History of abdominal surgery $[n(\%)]$ & & & \\
\hline
\end{tabular}

NOSES - natural orifice specimen extraction surgery group, $C L$ - conventional laparoscopic surgery group, BMI - body mass index.

Table III. Operation related variables

\begin{tabular}{|lccc|}
\hline Parameter & NOSES $(n=69)$ & $\mathrm{CL}(n=69)$ & $P$-value \\
\hline Operation time $[\mathrm{min}]$ & $241.18 \pm 75.57$ & $202.51 \pm 71.24$ & $<0.001$ \\
\hline Blood loss $[\mathrm{ml}]$ & $95.13 \pm 56.14$ & $98.60 \pm 52.63$ & 0.301 \\
\hline No. of lymph nodes & $16.58 \pm 7.44$ & $16.36 \pm 7.95$ & 0.721 \\
\hline Positive surgical margin & 0 & 0 & - \\
\hline Tumor rupture & 0 & 0 & - \\
\hline
\end{tabular}

NOSES - natural orifice specimen extraction surgery group, $C L$ - conventional laparoscopic surgery group.

nificantly lower than the values of $1.46 \pm 2.74,1.03$ \pm 1.78 and $0.91 \pm 1.56$ in the $\mathrm{CL}$ group $(p=0.017$, $p=0.030, p=0.046$, respectively), and there was no significant difference in perianal operations and obstetric history between the two groups. As the time went on, there was no difference in Wexner scores between the two groups at 12, 18 and 24 months after surgery $(p>0.05)$. Meanwhile, there was also a difference in the patients with Wexner scores $\geq 3$ (non-minor incontinence) in the two groups 1 month after surgery ( 22 vs. $12, p=0.048$ ). Due to the death of some patients, the questionnaires were not fully completed at 18 and 24 months (Table $V$ for details).

Follow-up data are presented in Table VI. In the NOSES group and CL group, there were 10 and 9 pa- tients lost to follow-up, respectively. In addition, 49 and 50 people in the two groups were followed for more than 60 months, respectively. The mean follow-up periods were $81.02 \pm 23.9$ and $81.75 \pm 23.70$ months and the median follow-up periods were 88.5 (25-112) and 88 (24-112) months, respectively $(p=$ 0.873) (Table VI). The Kaplan-Meier plots showed that the two groups had similar OS $(p=0.863)$ (Figure 1 ) and DFS $(p=0.961)$ (Figure 2). The 5-year and 8-year OS and DFS of patients as well as recurrence type in the two groups were also comparable $(p>0.05)$.

\section{Discussion}

Over the course of 30 years, minimally invasive surgery has gained widespread popularity and lap- 
Table IV. Postoperative data

\begin{tabular}{|lccc|}
\hline Parameter & NOSES $(n=69)$ & $\mathrm{CL}(n=69)$ & $P$-value \\
\hline Bowel function recovery [days] & $3.21 \pm 1.38$ & $4.35 \pm 1.46$ & $<0.001$ \\
\hline Postoperative length of stay [days] & $10.18 \pm 3.27$ & $13.65 \pm 5.69$ & $<0.001$ \\
\hline \begin{tabular}{l} 
Complications [ $n$ (\%)]: \\
\hline Incisional infection
\end{tabular} & $9(13.04)$ & $23(33.33)$ & 0.047 \\
\hline Incisional hernia (any time after surgery) & 0 & 6 & 0.028 \\
\hline Anastomotic leakage & 3 & 1 & 1.000 \\
\hline Pulmonary infection & 2 & 5 & 1.000 \\
\hline Urinary tract infection & 2 & 3 & 0.441 \\
\hline Deep venous thrombosis & $2(2.90)$ & $3(4.35)$ & 1.000 \\
\hline Preventative ileostomy [ $n$ (\%)] & & 0.681 \\
\hline Pain Score: & $3.91 \pm 1.63$ & $5.09 \pm 1.92$ & 0.441 \\
\hline Postoperative day 1 & $2.64 \pm 1.35$ & $3.71 \pm 2.62$ & $<0.001$ \\
\hline Postoperative day 3 & $1.99 \pm 1.03$ & $2.13 \pm 1.28$ & $<.001$ \\
\hline Postoperative day 7 & $4.17 \pm 1.25$ & $9.05 \pm 3.24$ & 0.217 \\
\hline Total scar length [cm] (3 month after surgery) & & $<0.001$ \\
\hline
\end{tabular}

NOSES - natural orifice specimen extraction surgery group, $C L$-conventional laparoscopic surgery group.

Table V. Anal function related data

\begin{tabular}{|c|c|c|c|}
\hline Parameter & NOSES $(n=69)$ & $\mathrm{CL}(n=69)$ & $P$-value \\
\hline Other perianal operations & $2(2.90)$ & $4(5.80)$ & 0.681 \\
\hline Childbirths $(n=32)$ & $1.81 \pm 0.77$ & $1.84 \pm 0.62$ & 0.630 \\
\hline \multicolumn{4}{|l|}{ Wexner scores: } \\
\hline Preoperative & $0.69 \pm 1.31$ & $0.66 \pm 1.34$ & 0.604 \\
\hline Wexner scores $\geq 3(n)$ & 6 & 5 & 0.753 \\
\hline 1 month after surgery & $2.57 \pm 3.05$ & $1.46 \pm 2.74$ & 0.017 \\
\hline Wexner scores $\geq 3(n)$ & 22 & 12 & 0.048 \\
\hline 3 months after surgery & $1.72 \pm 1.92$ & $1.03 \pm 1.78$ & 0.030 \\
\hline Wexner scores $\geq 3(n)$ & 16 & 9 & 0.122 \\
\hline 6 months after surgery & $1.28 \pm 1.62$ & $0.91 \pm 1.56$ & 0.046 \\
\hline Wexner scores $\geq 3(n)$ & 12 & 7 & 0.217 \\
\hline 12 months after surgery & $0.83 \pm 1.45$ & $0.78 \pm 1.51$ & 0.252 \\
\hline Wexner scores $\geq 3(n)$ & 8 & 6 & 0.573 \\
\hline 18 months after surgery & $0.79 \pm 1.43(n=68)$ & $0.81 \pm 1.60(n=68)$ & 0.767 \\
\hline Wexner scores $\geq 3(n)$ & 8 & 8 & 1.000 \\
\hline 24 months after surgery & $0.81 \pm 1.52(n=67)$ & $0.83 \pm 1.49(n=65)$ & 0.824 \\
\hline Wexner scores $\geq 3(n)$ & 9 & 7 & 0.595 \\
\hline
\end{tabular}

NOSES - natural orifice specimen extraction surgery group, $C L$ - conventional laparoscopic surgery group.

aroscopic colorectal surgery has been widely used [13-18]. In CL colorectal resection, an abdominal assisted incision of several centimeters reduces the minimally invasive effects of laparoscopic surgery and increases the chance of incisional hernia and incisional infection [18]. Severe pain is also a huge handicap for postoperative recovery by prolonging the bedridden time and interfering with the func- 
Table VI. Follow-up data

\begin{tabular}{|c|c|c|c|}
\hline Parameter & NOSES $(n=69)$ & $\mathrm{CL}(n=69)$ & $P$-value \\
\hline Follow-up period [months] & $81.02 \pm 23.93$ & $81.75 \pm 23.70$ & 0.873 \\
\hline Median [months] & 88.5 & 88 & - \\
\hline Range [months] & $25-112$ & 24-112 & - \\
\hline Lost to follow-up & 10 & 9 & 1.000 \\
\hline 5-year overall survival (\%) & 85.90 & 86.10 & - \\
\hline 5-year disease-free survival (\%) & 79.10 & 79.20 & - \\
\hline 8-year overall survival (\%) & 75.00 & 72.40 & - \\
\hline 8-year disease-free survival (\%) & 73.10 & 71.70 & - \\
\hline Type of recurrence $[n(\%)]$ : & & & 0.483 \\
\hline Locoregional relapse & $2(2.90)$ & $3(4.35)$ & \\
\hline Liver metastasis & $11(15.94)$ & $8(11.59)$ & \\
\hline Lung metastasis & $2(2.90)$ & $2(2.90)$ & \\
\hline Peritoneal metastasis & $2(2.90)$ & $4(5.80)$ & \\
\hline Other & 0 & 0 & \\
\hline
\end{tabular}

NOSES - orifice specimen extraction surgery group, $C L$ - conventional laparoscopic surgery group.

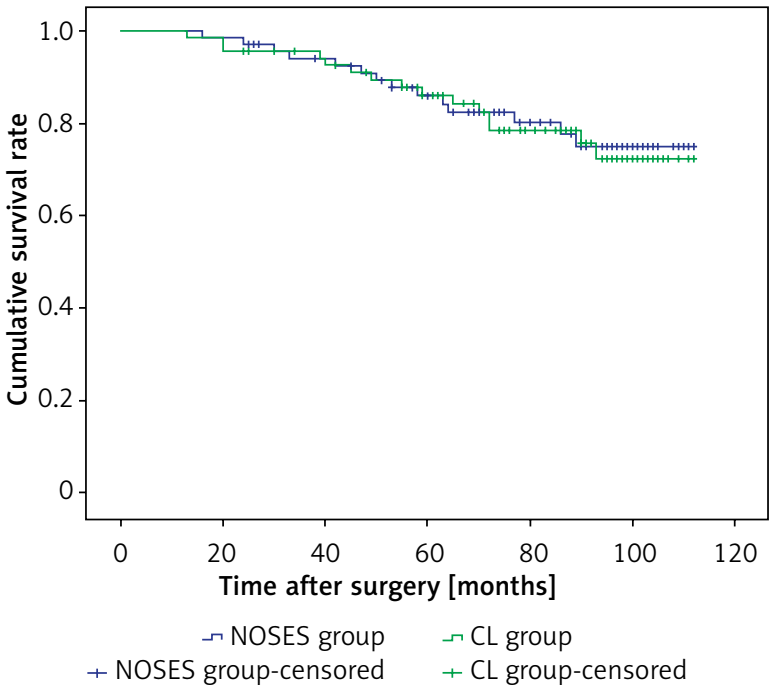

Figure 1. Comparison of overall survival rates between two groups ( $p=0.863$ )

NOSES - natural orifice specimen extraction surgery group, $C L$ - conventional laparoscopic surgery group.

tional recovery of multiple systems [19]. Associated complications may occur, such as respiratory tract infection, urogenital tract infection, deep vein thrombosis and even pressure ulcers [20]. In addition, the psychological effects of surgical scars should not be ignored. With the development of laparoscopic instruments and surgical techniques, abdominal surgery without an incision has become a new direction

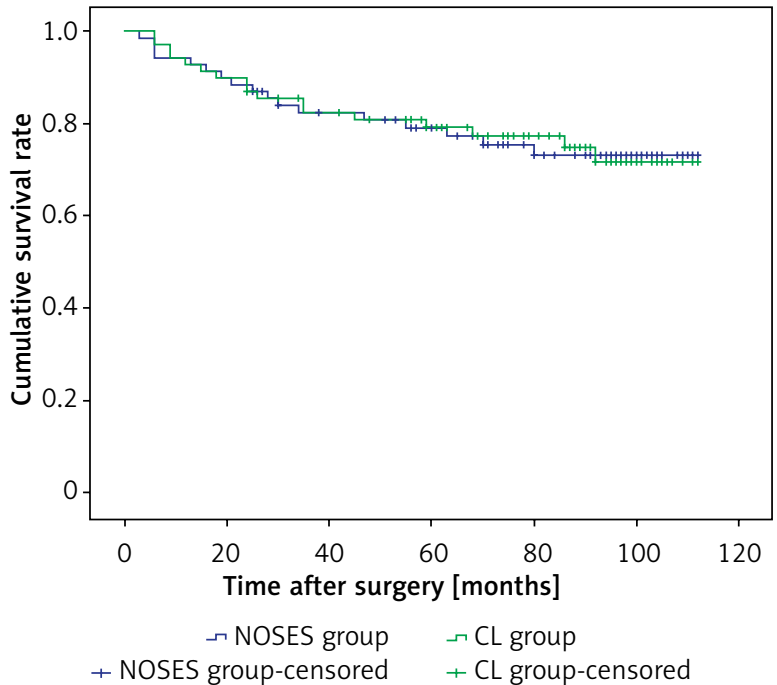

Figure 2. Comparison of disease-free survival rates between two groups ( $p=0.961$ )

NOSES - natural orifice specimen extraction surgery group, $C L$ - conventional laparoscopic surgery group.

of laparoscopic surgery [19]. NOSES eliminates the need of the abdominal assisted incision for specimen extraction, which is expected to reduce postoperative pain and some postoperative complications $[21,22]$.

This study proved that compared with $\mathrm{CL}$ surgery, NOSES has the advantages of faster recovery of intestinal function, shorter postoperative length 
of stay, lower incidence of incisional infection, less postoperative pain, and better cosmetic effect. The results are similar to those reported in the past $[1,3$, $4,7]$. Additionally, transanal NOSES colorectal cancer resection achieved similar efficacy of radical resection to $C L$ surgery, including adequate number of lymph node dissection, enough negative margin and similar long-term outcomes.

The results of Wexner scores have shown that transanal NOSES indeed impairs anal function and leads to fecal incontinence in more patients. With the passage of time, anal function can be restored to the same level as CL surgery, suggesting that the damage was generally reversible. However, the gap took 1 year to disappear, so the potential damage of anal function due to transanal NOSES should not be overlooked or hidden. For some patients, the benefits of NOSES may be negligible compared to a year of incontinence. In the NOSES group, there were 11 patients whose specimens did not go well in the extraction process and required repeated attempts. A narrow anus, poor elasticity of the anal sphincter, large tumor size, thick mesentery and obesity may prevent removal of the specimen via the anus. Forced extraction of the specimen can result in damage to the anal sphincter function. The procedure requires accurate evaluation of the matching degree between the anal sphincter and specimen before and during surgery, and appropriate selection of patients who may benefit from NOSES. The specimen should be removed gently and paroline can be used to reduce friction between the nylon bag and anus. The Wexner scores of patients in both groups did not return to their preoperative levels 2 years after surgery. Surgical trauma, decreased intestinal absorption function, radiotherapy and chemotherapy, as well as the increase of age, might affect the anal function $[10,23,24]$. Because normal aging affects anal function, we did not follow up anal function for longer periods of time than 2 years.

During NOSES colorectal resection, the sites of specimen extraction are usually the anus and vagina, which can be selected according to the patient's gender, fertility, tumor diameter and individual wishes [25]. There were some patients in our center who underwent transvaginal NOSES cancer resection, but most of them had the tumor in the right hemicolon. In practice, transvaginal NOSES is more acceptable to patients for the resection of right hemicolon cancer, since it could avoid enterotomy of the rectum during extraction and intracorporeal anastomosis. Since the operation procedure and functional outcomes of low rectal cancer are significantly different from those of middle and high rectal cancer [26], the lower margin of a tumor $<5 \mathrm{~cm}$ from the anal margin was taken as one of the exclusion criteria in this study.

Postoperative follow-up is important for patients with tumors, and an advantage of this study is the extremely long follow-up time. Some patients did not live in Beijing and could not attend appointments for examination and further treatment and some patients had changed their contact information, leading to some loss to follow-up. But the median time of 88.5 and 88 months is the longest reported follow-up time at present.

There are some limitation in this study. First of all, the small sample retrospective study lacks statistical power to reach a strong conclusion. In addition, it would be reasonable to preform a long-term overall quality of life assessment including sexual function and level of activities. However, due to the limited database, we cannot provide more information on quality of life other than Wexner fecal incontinence scores. Further prospective, randomized, controlled studies with comprehensive evaluation of postoperative quality of life are needed.

\section{Conclusions}

This study showed that transanal NOSES for sigmoid colon or rectal cancer resection achieves similar long-term outcomes for patients with colorectal cancer to those achieved by $\mathrm{CL}$ surgery. Compared with CL surgery, transanal NOSES has the advantages of faster recovery of intestinal function, shorter postoperative length of stay, less incisional pain, lower incisional infection rate, and a better cosmetic effect. However, transanal NOSES indeed impairs anal function, needing more attention.

\section{Acknowledgments}

Jianlu Zhang and Weiqing Li contributed equally to this study. Zhipeng Sun (sunzhipeng@bjsjth.cn) and Bin Zhu (zhubin@bjsjth.cn, binbinzhu99@sohu. com) contributed equally to this study.

\section{Conflict of interest}

The authors declare no conflict of interest. 


\section{References}

1. Guan X, Lu Z, Wang S, et al. Comparative short- and long-term outcomes of three techniques of natural orifice specimen extraction surgery for rectal cancer. Eur J Surg Oncol 2020; 46: e55-61.

2. Wolthuis AM, Fieuws S, Van Den Bosch A, et al. Randomized clinical trial of laparoscopic colectomy with or without natural-orifice specimen extraction. Br J Surg 2015; 102: 630-7.

3. Wolthuis AM, de Buck van Overstraeten A, D’Hoore A. Laparoscopic natural orifice specimen extraction-colectomy: a systematic review. World J Gastroenterol 2014; 20: 12981-92.

4. Hisada M, Katsumata K, Ishizaki T, et al. Complete laparoscopic resection of the rectum using natural orifice specimen extraction. World I Gastroenterol 2014; 20: 16707-13.

5. He J, Hu JF, Shao SX, et al. The comparison of laparoscopic colorectal resection with natural orifice specimen extraction versus mini-laparotomy specimen extraction for colorectal tumours: a systematic review and meta-analysis of short-term outcomes. J Oncol 2020; 2020: 6204264.

6. Awad ZT, Griffin R. Laparoscopic right hemicolectomy: a com parison of natural orifice versus transabdominal specimen extraction. Surg Endosc 2014; 28: 2871-6.

7. Zhou S, Wang X, Zhao C, et al. Comparison of short-term and survival outcomes for transanal natural orifice specimen extraction with conventional mini-laparotomy after laparoscopic anterior resection for colorectal cancer. Cancer Manag Res 2019; 11: 5939-48.

8. Leroy J, Costantino F, Cahill RA, et al. Laparoscopic resection with transanal specimen extraction for sigmoid diverticulitis. Br J Surg 2011; 98: 1327-34.

9. Costantino FA, Diana M, Wall J, et al. Prospective evaluation of peritoneal fluid contamination following transabdominal vs. transanal specimen extraction in laparoscopic left-sided colorectal resections. Surg Endosc 2012; 26: 1495-500.

10. Jakubauskas M, Jotautas V, Poskus E, et al. Fecal incontinence after transanal endoscopic microsurgery. Int I Colorectal Dis 2018; 33: 467-72

11. Jorge JM, Wexner SD. Etiology and management of fecal incontinence. Dis Colon Rectum 1993; 36: 77-97.

12. Hayden DM, Weiss EG. Fecal incontinence: etiology, evaluation, and treatment. Clin Colon Rectal Surg 2011; 24: 64-70.

13. Biatecki JT, Myszka W, Wysocka E, et al. A comparison of the oxidative stress response in single-incision laparoscopic versus multi-trocar laparoscopic totally extraperitoneal inguinal hernia repair. Videosurgery Miniinv 2020; 15: 567-73.

14. Ma J, Yang J, Cheng S, et al. Prediction model of laparoendoscopic single-site surgery in gynecology using machine learning algorithm. Videosurgery Miniinv 2021; 16: 587-96.

15. Jacobs M, Verdeja JC, Goldstein HS. Minimally invasive colon resection (laparoscopic colectomy). Surg Laparosc Endosc 1991; 1: $144-150$.

16. Bonjer HJ, Deijen CL, Haglind E; COLOR II Study Group. A randomized trial of laparoscopic versus open surgery for rectal cancer. N Engl J Med 2015; 373: 194.

17. Guillou PJ, Quirke P, Thorpe H, et al. Short-term endpoints of conventional versus laparoscopic-assisted surgery in patients with colorectal cancer (MRC CLASICC trial): multicentre, randomised controlled trial. Lancet 2005; 365: 1718-26.

18. Parker JM, Feldmann TF, Cologne KG. Advances in laparoscopic colorectal surgery. Surg Clin North Am 2017; 97: 547-60.

19. Lee L, Mappin-Kasirer B, Sender Liberman A, et al. High incidence of symptomatic incisional hernia after midline extraction in laparoscopic colon resection. Surg Endosc 2012; 26: 3180-5.

20. Leroy J, Barry BD, Melani A, et al. No-scar transanal total mesorectal excision: the last step to pure NOTES for colorectal surgery. JAMA Surg 2013; 148: 226-31.

21. Han Y, He YG, Zhang HB, et al. Total laparoscopic sigmoid and rectal surgery in combination with transanal endoscopic microsurgery: a preliminary evaluation in China. Surg Endosc 2013; 27: 518-24.

22. Clark MP, Qayed ES, Kooby DA, et al. Natural orifice translumenal endoscopic surgery in humans: a review. Minim Invasive Surg 2012; 2012: 189296.

23. Restivo A, Zorcolo L, D'Alia G, et al. Risk of complications and long-term functional alterations after local excision of rectal tumors with transanal endoscopic microsurgery (TEM). Int J Colorectal Dis 2016; 31: 257-66.

24. Nevler A. The epidemiology of anal incontinence and symptom severity scoring. Gastroenterol Rep 2014; 2: 79-84.

25. Guan X, Liu Z, Longo A, et al. International consensus on natural orifice specimen extraction surgery (NOSES) for colorectal cancer. Gastroenterol Rep 2019; 7: 24-31.

26. Marks JH, Salem JF, Valsdottir EB, et al. Quality of life and functional outcome after transanal abdominal transanal proctectomy for low rectal cancer. Dis Colon Rectum 2017; 60: 258-65.

Received: 6.10.2021, accepted: 5.12.2021 